\title{
Collagenase Clostridium Histolyticum Injection for Dupuytren Contracture: 2-Year Follow-up
}

\author{
Suresh K. Nayar, MD, Dennis Pfisterer, DO*, John V. Ingari, MD \\ Department of Orthopaedic Surgery, Johns Hopkins University, Baltimore, MD, \\ ${ }^{*}$ WellSpan York Hospital, York, PA, USA
}

\begin{abstract}
Background: Dupuytren disease is characterized by the development of palmar fibrous tissue that can lead to fixed flexion contracture (FFC) and contribute to functional loss of the involved digits. Our goal was to investigate rates of contracture resolution and recurrence in patients who underwent enzymatic fasciotomy for Dupuytren contracture consisting of collagenase clostridium histolyticum (CCH) injection followed by passive manipulation combined with splinting and home-based therapy.

Methods: We prospectively enrolled 34 patients (44 metacarpophalangeal [MCP] and 33 proximal interphalangeal [PIP] joints) treated by one orthopaedic hand surgeon between November 2010 and November 2014. On day 1, CCH was injected into a palpable fibrous cord of the involved fingers. The next day, the finger was passively extended to its maximal corrective position. FFC was measured for each joint before injection and immediately after manipulation. Patients were instructed to wear an extension splint at night and perform stretching exercises at home and were re-evaluated at 6 weeks, 4 months, 1 year, and 2 years. Resolution was defined as improvement of contracture to $\leq 5^{\circ}$ of neutral. Recurrence was defined as an increase in FCC of $\geq 20^{\circ}$ after treatment.
\end{abstract}

Results: Immediate contracture resolution occurred in 42 of $44 \mathrm{MCP}$ joints $(p<0.001)$, improving from $50^{\circ}$ to $1.5^{\circ}$, and in 14 of 33 PIP joints $\left(p=0.182\right.$ ), improving from $44^{\circ}$ to $16^{\circ}$. Four joints had recurrence within 6 weeks. Of the 48 joints with minimum 4-month follow-up (mean, 26 months), 12 had recurrence at 2-year follow-up (MCP, 6; PIP, 6). At 2-year follow-up, MCP and PIP contractures measured $17^{\circ}$ and $35.5^{\circ}$, respectively. Older age and multiple digit involvement were associated with higher recurrence rates.

Conclusions: CCH offers a safe, nonoperative option to correct FCC in Dupuytren disease with greater success for MCP joints compared to PIP joints. There is a tendency of reoccurrence within 2 years of treatment. Further investigation is needed to determine optimal timing of repeat $\mathrm{CCH}$ injection to improve upon or extend the period of contracture resolution.

Keywords: Dupuytren contracture, Collagenase clostridium histolyticum, Fixed flexion contractures, Metacarpophalangeal joint, Proximal interphalangeal joint

Dupuytren contracture is characterized by the development of fibrous tissue in the form of nodules and cords within the palmar fascia. This process has features similar to wound-healing tissue, with associated contraction and

Received March 11, 2019; Revised April 16, 2019; Accepted April 26, 2019 Correspondence to: Suresh K. Nayar, MD

Department of Orthopaedic Surgery, Johns Hopkins University, 601 North Caroline St, Baltimore, MD 21287, USA

Tel: +1-443-997-2663, Fax: +1-410-502-6816

E-mail: snayar2@jhmi.edu maturation. ${ }^{1,2)}$ The process occurs in the fibro-fatty layer between the skin and deep structures of the palmar surface of the hand, which has a precisely ordered network of subcutaneous ligamentous fibers. The abnormal fibrous Dupuytren tissue develops within and/or around these longitudinally oriented ligamentous fibers. ${ }^{3)}$

Nodules and cords develop at precise locations, typically in line with the digital rays in the palm and/or fingers. The disease appears to follow tension lines within the palmar fascia. Nodules may later mature to form longitudinally oriented cords. The normal palmar fascia and cord 
form a diseased band of tissue with thickening in response to increased tension. As the fibrous tissue matures and contracts, patients develop progressive flexion contractures of the hand and fingers. Over time, this contributes to a progressive loss of function in the involved extremity.

In the past, emphasis has been placed on surgical treatment. Outcomes have been highly variable because they depend on the integrated treatment plan consisting of surgical technique selection, timing of surgery, patient expectations, and postoperative rehabilitation program. ${ }^{1,45}$ Surgical treatment carries major risks including injury to digital nerves or vessels, resultant proximal interphalangeal (PIP) joint hyperextension, and wound healing complications related to hematoma, skin necrosis, and infection. ${ }^{5}$ Surgical treatment may also result in recurrent stiffness, complex regional pain syndrome, and stiffness elsewhere in the affected hand. ${ }^{3,4)}$

In February 2010, the United States Food and Drug Administration approved collagenase clostridium histolyticum (CCH) (Xiaflex; Auxilium Pharmaceuticals Inc., Malvern, PA, USA) as a nonoperative treatment for Dupuytren contracture. CCH contains two microbial collagenases in a defined mass ratio: collagenase AUX-I and collagenase AUX II, which are isolated and purified from the fermentation of Clostridium histolyticum bacteria. This nonsurgical option can be performed in an office setting and was designed to avoid complications associated with surgery. Initial studies showed promising results and minimal morbidity after in-office injection. ${ }^{3-6)}$ Furthermore, this nonoperative option does not require the extensive physical therapy that is needed after surgery to prevent stiffness and maintain correction.

The purpose of this study was to examine the rates of contracture resolution and recurrence in patients who underwent enzymatic fasciotomy consisting of $\mathrm{CCH}$ injection, manipulation, splinting, and home-based therapy for treatment of Dupuytren contracture involving the metacarpophalangeal (MCP) or PIP joint. Given the successful maintenance of contracture resolution described by multiple studies at 1 year $^{7-10)}$ and the tendency for recurrence during longer follow-up periods, ${ }^{11-13)}$ we hypothesized that contracture resolution would be maintained at 1 year but would tend to return by 2 years after $\mathrm{CCH}$ injection.

\section{METHODS}

\section{Patient Selection}

After Institutional Review Board approval, 34 patients (10 women) were enrolled in this prospective trial between November 2010 and November 2014. Enrolled patients were treated by one fellowship-trained, orthopaedic hand surgeon at a community hospital. We included patients with symptomatic Dupuytren contracture (with functional limitation) with a palpable cord and fixed flexion contracture (FFC) $\geq 30^{\circ}$ at the MCP joint and/or PIP joint of any digit other than the thumb who preferred nonoperative treatment. We excluded patients for the following reasons: previous treatment for FFC (both surgical and nonoperative), previous hand trauma resulting in scarring deformity, documented neurological and/or musculoskeletal disorder as a cause of finger/hand contractures, current breast-feeding or pregnancy, bleeding disorders, recent stroke, or a neuromuscular disorder affecting the hands. Overall, 77 joints were treated (44 MCP and 33 PIP joints). The mean patient age was 71 years. Consent was obtained in all cases.

\section{Treatment}

Initial treatment involved a 2-day process. Before injection (on day 1), FFCs were measured by the same fellowshiptrained hand surgeon or a senior resident under direct supervision using a standard goniometer. Xiaflex $(0.9 \mathrm{mg}$ of $\mathrm{CCH}$ ) was injected using a 27 -gauge, $12-\mathrm{mm}$ length needle at three sites $2-3 \mathrm{~mm}$ apart along each affected cord. All patients received the same total volume of $\mathrm{CCH}$ per digit. On day 2 (the next day), the affected finger was anesthetized with $1 \%$ lidocaine and then manipulated by passively extending it to its maximal corrective position. Moderate and steady stretching pressure was applied while slowly extending the finger for 10- to 20-second intervals for a maximum of three attempts, each spaced 5 minutes apart. We avoided jerking the finger with sudden movement to avoid tendon rupture. At this point, contracture was measured again, as on day 1 . After manipulation, the finger was placed in an extension splint for night use. Skin tears associated with manipulation were treated with local wound care. We evaluated the maintenance of correction at 6 weeks, 4 months, 1 year, and 2 years after the procedure. No patient in this series underwent surgical treatment during the duration of this study.

\section{Physical Therapy}

The physical therapy protocol then started with extension splinting at night for up to 4 months if residual contracture had not corrected by 6 weeks. During the day, patients performed both active and passive finger/hand stretching exercises at home, which were taught to each patient by a certified hand therapist. These exercises included lifting each finger individually off a flat surface holding full extension for 5 seconds; abducting and adducting each fin- 
ger in a windshield wiper motion; and extending the MCP joints while holding the distal and PIP joints in full flexion. Each exercise was repeated ten times per finger four times a day up to 6 weeks. Patients were instructed to wear their extension splint during the day when not performing these exercises.

\section{Statistical Analysis}

Chi-square testing was used to determine successful initial treatment and recurrence for all joints. Resolution was defined as improvement of contracture to $\leq 5^{\circ}$ of neutral. Recurrence was defined as an increase in FCC of $\geq 20^{\circ}$ after treatment. Nonparametric $t$-tests were used to compare median FFCs at baseline and 2-year follow-up.

\section{RESULTS}

\section{Immediate Contracture Resolution}

The mean preinjection contracture for all 77 joints was $47^{\circ}$ (50 ${ }^{\circ}$ for MCP and $44^{\circ}$ for PIP joints). After manipulation, on the day after $\mathrm{CCH}$ injection (day 2 ), the mean residual contracture was $7.7^{\circ}$ for all joints $\left(1.5^{\circ}\right.$ for MCP and $16^{\circ}$ for PIP joints). Immediate postmanipulation FCC resolution was recorded in 56 joints (42/44 MCP and 14/33 PIP joints) on day 2 . Of the 21 joints that did not correct to $\leq 5^{\circ}$, 19 involved the PIP joint. Preinjection contracture severity for MCP joints had no effect on postmanipulation resolution. While more severe preinjection contracture involving PIP joints trended toward incomplete contracture resolu-

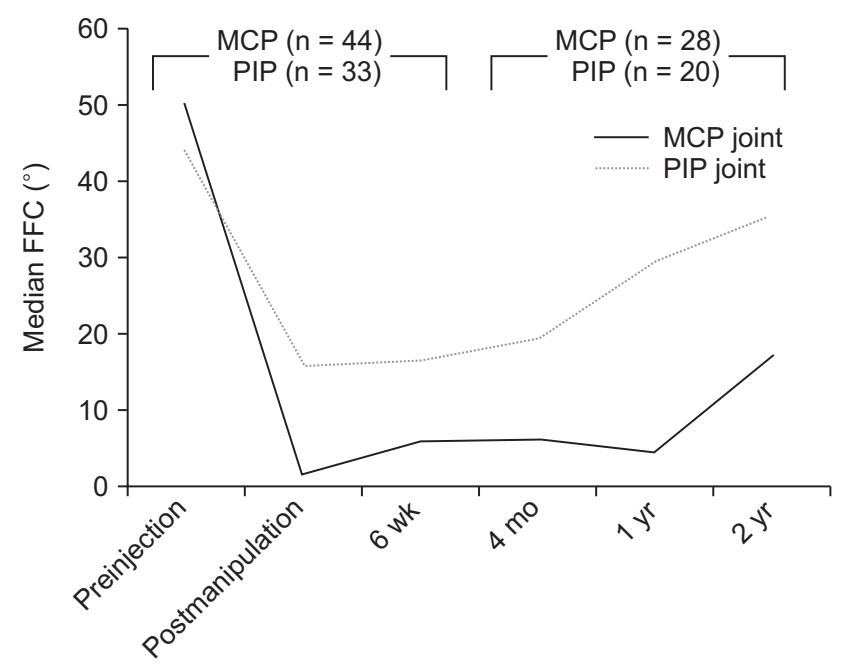

Fig. 1. Fixed flexion contracture (FFC) measurement for 77 joints after collagenase clostridium histolyticum injection. Only 48 joints were available for follow-up after the 4-month evaluation period. MCP: metacarpophalangeal, PIP: proximal interphalangeal. tion, this was not an appreciable relationship.

\section{Maintenance of Contracture Resolution}

At 6-week follow-up, the mean residual contracture had increased from $7.7^{\circ}$ to $10^{\circ}$. The mean contractures of the MCP and PIP joints were $5.5^{\circ}$ and $17^{\circ}$, respectively. At 4-month, 1-year, and 2-year follow-up visits, the FFC gradually increased in both joints, affecting the PIP more so than the MCP joint (Fig. 1). At 2-year follow-up, the mean FFCs for the MCP and PIP joints were $17^{\circ}$ and $35.5^{\circ}$, respectively. Although a significant contracture improvement for MCP joints remained from pretreatment to 2 years $(p<0.001)$, there was no improvement for PIP joints during the same period $(p=0.642)$.

\section{Contracture Recurrence}

The rate of early recurrence, within the first 6 weeks, was $5.2 \%(4 / 77)$. Forty-eight joints had long-term follow-up (at least 4 months after treatment). This represented $28 \mathrm{MCP}$ and 20 PIP joints, with a mean follow-up of 26 months. Of the 48 joints available at 4-month follow-up, there were 12 recurrences, for a success rate of $75 \%(36 / 48, p=0.001)$. Of the 12 recurrences, six were in MCP joints, and six were in PIP joints. Six of the patients who experienced FFC recurrence had multiple fingers and joints involved. Their mean age was 75 years.

\section{Complications}

There were only minor complications observed in our patient cohort. There were no iatrogenic flexor tendon ruptures. Nine patients had skin tears during manipulation. All were treated successfully with Epsom salt soaks and local wound care until they healed. No infections were noted in our study group. There was one small finger nerve neuropraxia, which was asymptomatic but had not resolved at the final follow-up visit.

\section{DISCUSSION}

In this small, community hospital patient cohort, there was near-complete immediate contracture resolution in 42 of 44 of MCP joints (95\%) and 14 of 33 PIP joints (42\%). This selective improvement of MCP contracture is likely attributable to the fact that cords proximal to the MCP joint are often more distinct and palpable compared with cords distal to the MCP joint. For the 48 joints that experienced contracture resolution and were available for 2-year follow-up, 12 (six MCP and six PIP) experienced contracture recurrence. In general, we observed the majority of FFC progression to occur after the 4-month follow-up 
visit for PIP joints and after the 1-year follow-up visit for MCP joints. Given these results, within the first and 2-year follow-up periods may be an important time to reconsider repeat injection and manipulation for PIP and MCP joints, respectively. Finally, although the overall mean FFC increase did not reach our definition of recurrence $\left(\geq 20^{\circ}\right.$ increase), there was a gradual contracture increase during the follow-up period, which is likely to increase even further over time.

During the past several years, studies with shortterm $(<3 \text { months })^{14-19)}$ or 1-year ${ }^{7-10,20)}$ results have reported promising FFC resolution after $\mathrm{CCH}$ injection. Longerterm studies have also shown similar resolution rates and contracture improvement but showed mixed results with recurrence. One study including 57 patients with 2-year follow-up showed FFC improvement for MCP joints (54 to $9^{\circ}$ ) and PIP joints $\left(30^{\circ}\right.$ to $\left.16^{\circ}\right)$, with complete resolution in $80 \%$ of MCP joints and in less than half of PIP joints. ${ }^{11)}$ Another 2-year study with 87 patients showed improvement from $39^{\circ}$ to $14^{\circ}$ in MCP joints and from $54^{\circ}$ to $32^{\circ}$ in PIP joints. ${ }^{21)}$ In a third study of 47 patients with 2-year follow-up, isolated MCP cords showed improvement from $42^{\circ}$ to $11^{\circ} .{ }^{12)}$ Our study reports rates of resolution and magnitude of correction of FFC that are similar to those of these 2-year follow-up studies.

Multiple reviews have shown the benefits of $\mathrm{CCH}$ injection but limited follow-up periods and high recurrence rates should be noted. ${ }^{22,23)}$ One study with 25 patients with a longer-term follow-up (32 months) showed contracture improvement from $43.5^{\circ}$ to $3.6^{\circ}$ in MCP joints and from $30.0^{\circ}$ to $17.5^{\circ}$ in PIP joints. ${ }^{24)}$ These results are superior to those cited in 2-year follow-up studies; however, it should be noted that the sole author of the study was a consultant for Auxilium Pharmaceuticals, the parent company of Xiaflex. One of the largest studies to assess the long-term efficacy of clostridium injection was the CORDLESS trial, which reported 5-year follow-up data for 623 joints. ${ }^{13)}$ At 3-year follow-up, the authors reported recurrence (defined as $\geq 20^{\circ}$ in FFC) rates of $16 \%$ for MCP joints and 38\% for PIP joints, which increased to 39\% and $66 \%$ by 5 years, respectively. Compared with other methods of treating Dupuytren contracture, such as needle or open fasciotomy, the authors noted lower complications rates for neurovascular injury and complex regional pain syndrome with equal efficacy in contracture resolution and recurrence.

Our study has limitations. We included only 34 patients in our study with some loss to follow-up; however, the rates of recurrence and degree of FFC resolution were similar to those of other studies at 1- and 2-year follow-up. While this was not a large multi-center study, we were able to assess our patients with greater granularity and under the care of a single surgeon, eliminating heterogeneity introduced by different providers. The definitions of recurrence ( $\geq 20^{\circ}$ increase in FFC from baseline manipulation) and "successful" contracture resolution $\left(\leq 5^{\circ}\right.$ residual contracture) are arbitrary and are reported in a manner consistent with previous studies, which may under-represent patient-perceived success of treatment. For example, if an initial contracture of $70^{\circ}$ was corrected to $10^{\circ}$, this would be an unsuccessful manipulation according to our definition; yet, the patient might be satisfied with this improvement.

In conclusion, $\mathrm{CCH}$ injection for Dupuytren contracture is a viable nonoperative option for contracture resolution, showing greater improvement for contractures of the MCP joint compared with the PIP joint. Similar to previous studies, we report a tendency for contractures to recur over time. Further investigation is needed to determine optimal timing of repeat $\mathrm{CCH}$ injection to improve upon or extend the period of contracture resolution.

\section{CONFLICT OF INTEREST}

No potential conflict of interest relevant to this article was reported.

\section{ACKNOWLEDGEMENTS}

We would like to thank Ms. Rachel Box, MS, Ms. Jenni Weems, MS, and Ms. Kerry Kennedy, BA (Department of Orthopaedics, Johns Hopkins University School of Medicine) for manuscript preparation and editorial services.

\section{REFERENCES}

1. Wolfe SW, Hotchkiss RN, Pederson WC, Kozin SH, Cohen MS. Green's operative hand surgery. 7th ed. Philadelphia, PA: Elsevier; 2017. 128-49.

2. Tomasek J, Rayan GM. Correlation of alpha-smooth muscle actin expression and contraction in Dupuytren's disease fibroblasts. J Hand Surg Am. 1995;20(3):450-5.

3. Gilpin D, Coleman S, Hall S, Houston A, Karrasch J, Jones N. Injectable collagenase Clostridium histolyticum: a new 
nonsurgical treatment for Dupuytren's disease. J Hand Surg Am. 2010;35(12):2027-38.

4. Badalamente MA, Hurst LC. Efficacy and safety of injectable mixed collagenase subtypes in the treatment of Dupuytren's contracture. J Hand Surg Am. 2007;32(6):767-74.

5. Hurst LC, Badalamente MA, Hentz VR, et al. Injectable collagenase clostridium histolyticum for Dupuytren's contracture. N Engl J Med. 2009;361(10):968-79.

6. Rajesh KR, Rex C, Mehdi H, Martin C, Fahmy NR. Severe Dupuytren's contracture of the proximal interphalangeal joint: treatment by two-stage technique. J Hand Surg Br. 2000;25(5):442-4.

7. Binter A, Neuwirth M, Rab M. Treatment of Dupuytren's disease with collagenase: a 1-year follow-up of 37 patients. Handchir Mikrochir Plast Chir. 2014;46(6):355-60.

8. Keller M, Arora R, Schmiedle G, Kastenberger T. Treatment of Dupuytren's disease with collagenase Clostridium histolyticum. Orthopade. 2017;46(4):321-7.

9. Muppavarapu RC, Waters MJ, Leibman MI, Belsky MR, Ruchelsman DE. Clinical outcomes following collagenase injections compared to fasciectomy in the treatment of Dupuytren's contracture. Hand (N Y). 2015;10(2):260-5.

10. Odinsson A, Brenne LE, Lurie TB, Finsen V. Dupuytren's contracture: the safety and efficacy of collagenase treatment. J Hand Surg Asian Pac Vol. 2016;21(2):187-92.

11. Lauritzson A, Atroshi I. Collagenase injections for Dupuytren's disease: prospective cohort study assessing 2-year treatment effect durability. BMJ Open. 2017;7(3):e012943.

12. McFarlane J, Syed AM, Sibly TF. A single injection of collagenase clostridium histolyticum for the treatment of moderate Dupuytren's contracture: a 2 year follow-up of 47 patients. J Hand Surg Eur Vol. 2016;41(6):664-5.

13. Peimer CA, Blazar P, Coleman S, Kaplan FT, Smith T, Lindau T. Dupuytren contracture recurrence following treatment with collagenase clostridium histolyticum (CORDLESS [Collagenase Option for Reduction of Dupuytren Long-Term Evaluation of Safety Study]): 5-year data. J Hand Surg Am. 2015;40(8):1597-605.
14. Atroshi I, Strandberg E, Lauritzson A, Ahlgren E, Walden M. Costs for collagenase injections compared with fasciectomy in the treatment of Dupuytren's contracture: a retrospective cohort study. BMJ Open. 2014;4(1):e004166.

15. Considine S, Heaney R, Hirpara KM. Early results of the use of collagenase in the treatment of Dupuytren's contracture. Ir J Med Sci. 2015;184(2):323-7.

16. Sakai A, Zenke Y, Menuki K, et al. Short-term efficacy and safety of collagenase injection for Dupuytren's contracture: therapy protocol for successful outcomes in a clinical setting. J Orthop Sci. 2019;24(3):434-40.

17. Tay TK, Tien H, Lim EY. Comparison between collagenase injection and partial fasciectomy in the treatment of Dupuytren's contracture. Hand Surg. 2015;20(3):386-90.

18. Verheyden JR. Early outcomes of a sequential series of 144 patients with Dupuytren's contracture treated by collagenase injection using an increased dose, multi-cord technique. J Hand Surg Eur Vol. 2015;40(2):133-40.

19. Zhou C, Hovius SE, Slijper HP, et al. Collagenase clostridium histolyticum versus limited fasciectomy for Dupuytren's contracture: outcomes from a multicenter propensity score matched study. Plast Reconstr Surg. 2015;136(1):87-97.

20. Scherman P, Jenmalm P, Dahlin LB. One-year results of needle fasciotomy and collagenase injection in treatment of Dupuytren's contracture: a two-centre prospective randomized clinical trial. J Hand Surg Eur Vol. 2016;41(6):577-82.

21. Van Beeck A, Van den Broek M, Michielsen M, Didden K, Vuylsteke K, Verstreken F. Efficacy and safety of collagenase treatment for Dupuytren's disease: 2-year follow-up results. Hand Surg Rehabil. 2017;36(5):346-9.

22. Smeraglia F, Del Buono A, Maffulli N. Collagenase clostridium histolyticum in Dupuytren's contracture: a systematic review. Br Med Bull. 2016;118(1):149-58.

23. Soreide E, Murad MH, Denbeigh JM, et al. Treatment of Dupuytren's contracture: a systematic review. Bone Joint J. 2018;100(9):1138-45.

24. Naam NH. Functional outcome of collagenase injections compared with fasciectomy in treatment of Dupuytren's contracture. Hand (N Y). 2013;8(4):410-6. 